\title{
Seleção de rizóbios para o Trifolium repens em condições de solo alagado
}

\author{
Jonatas Bredow Alves ${ }^{1}$ \\ Enilson Luiz Saccol de Sá ${ }^{*}$ \\ Aleksander Westphal Muniz ${ }^{2}$ \\ ${ }^{1}$ PPG em Ciência do Solo, Laboratório de Microbiologia do Solo \\ Faculdade de Agronomia,Universidade Federal do Rio Grande do Sul \\ Avenida Bento Gonçalves, 7712, CEP, Porto Alegre-RS, Brasil \\ ${ }^{2}$ Empresa Brasileira de Pesquisa Agropecuária - Embrapa Amazônia Ocidental \\ *Autor para correspondência \\ aleksander.muniz@embrapa.br
}

Submetido em 28/03/2011

Aceito para publicação em 15/10/2011

\section{Resumo}

O estado do Rio Grande do Sul possui cerca de 5,4 milhões de hectares várzeas. A implantação de leguminosas forrageiras de inverno como trevo branco é uma ótima alternativa para aumentar a produtividade destas áreas por meio da fixação biológica de nitrogênio (FBN). Esse benefício pode ser obtido com associação da leguminosa a estirpes de rizóbio que fixem o nitrogênio de forma eficiente nessas condições. O objetivo do trabalho foi selecionar rizóbios eficientes na FBN para trevo branco, e avaliar a tolerância dessa simbiose em condições de alagamento do solo. Para isso, foi realizado um experimento em casa de vegetação em vasos com solo utilizando 14 isolados. Cada tratamento foi mantido sob duas condições de umidade do solo, com alagamento e próxima à capacidade de campo. $\mathrm{O}$ alagamento do solo reduziu a nodulação, a produção de matéria seca e o acúmulo de nitrogênio na parte aérea das plantas inoculadas com rizóbio. $\mathrm{O}$ efeito do alagamento do solo na FBN variou de acordo com o isolado avaliado. Os isolados CVII, $\mathrm{P}_{3} 4$, T4 e VP 16 apresentaram alta eficiência de fixação do nitrogênio em condições de alagamento do solo e superaram o tratamento controle com adição de nitrogênio mineral.

Palavras-chave: Estresse hídrico, Fixação biológica de nitrogênio, Rhizobium, Trevo Branco

\section{Abstract}

Selection of rhizobia for Trifolium repens in flooded soil conditions. The Brazilian state of Rio Grande do Sul has approximately 5.4 million hectares of lowland. The introduction of winter forage legumes, such as white clover, can increase the productivity of these lowland areas through biological nitrogen fixation (BNF). However, this benefit can only occur if the legume is associated with rhizobia that can efficiently fix nitrogen under available soil conditions. The objective of this study was to select rhizobia of Rio Grande do Sul soils, which are efficient in BNF when in symbiosis with white clover, and evaluate the tolerance of this symbiosis under flooded soil conditions. The experiment was conducted in a greenhouse, in soil-filled pots, where 14 isolates were evaluated for nitrogen fixation ability under flooded soil conditions. Each treatment was tested under two soil moisture conditions (flooded and field simulated). Flooding significantly reduced the number and nodule 
weight as well as the production of dry matter and nitrogen accumulation in the shoots of plants inoculated with rhizobia. The effect of soil flooding on BNF varied according the isolate evaluated. The strains CVII, $\mathrm{P}_{3} 4, \mathrm{~T} 4$ and VP16 exhibited high nitrogen fixation efficiency under flooding conditions, surpassing the nitrogen-added control treatment, on nitrogen accumulation in shoots.

Key words: Biological nitrogen fixation, Hydric stress, Rhizobium, White clover

\section{Introdução}

No estado do Rio Grande do Sul, existem cerca de 5,4 milhões de hectares de áreas de várzea, caracterizadas pelo relevo plano e pela presença de solos hidromórficos com drenagem natural deficiente, o que resulta em períodos de alagamento do solo nas épocas de maior precipitação pluviométrica (MARCHEZAN et al., 2002). Dessas áreas, cerca de um milhão de hectares são utilizados anualmente com o cultivo do arroz irrigado, sendo o restante basicamente utilizado pela pecuária extensiva, com baixos índices de produtividade animal (MARCHEZAN et al., 2002).

A implantação de pastagens de inverno seria uma excelente alternativa para o melhor aproveitamento dessas áreas, pois proporcionaria um aumento da lotação animal, aumentando os índices de produtividade animal e consequentemente, proporcionando um maior retorno econômico (MARCHEZAN et al., 2002). Porém, a grande maioria das plantas não é adaptada às condições de alagamento, como baixas concentrações de oxigênio no solo (VARTAPETIAN; JACKSON, 1997).

Além de prejudicar o crescimento de plantas, o alagamento do solo pode também reduzir a fixação simbiótica de nitrogênio, principalmente pela redução no suprimento de oxigênio para os nódulos radiculares (ZHANG; SMITH, 2002). Porém, o alagamento do solo pode não afetar negativamente a fixação simbiótica de nitrogênio, especialmente em plantas que apresentem a capacidade de formação de aerênquima e a emissão de raízes adventícias nessas condições (PUGH et al., 1995; JAMES; CRAWFORD, 1998; JAMES; SPRENT, 1999).

Por outro lado, algumas espécies do gênero Trifolium como o trevo branco (Trifolium repens L.), apresentam mecanismos de tolerância ao alagamento do solo, como a formação de aerênquima e a emissão de raízes adventícias (ROGER; WEST, 1993; GIBBERD et al., 2001). Além disso, o trevo branco apresenta outras características desejáveis como comportamento perene e alta capacidade de fixação simbiótica de nitrogênio (CARLSSON; HUSS-DANELL, 2003).

Desta forma, plantas de trevo branco, inoculadas com estirpes eficientes de Rhizobium leguminosarum biovar trifolii, podem ser capazes de fixar nitrogênio em condições de alagamento do solo. Além disso, é possível que nessas condições exista variabilidade na eficiência de fixação de nitrogênio por diferentes isolados de rizóbio. O presente trabalho teve como objetivo selecionar rizóbios eficientes na fixação de nitrogênio em simbiose com trevo branco, em condições de alagamento do solo, assim como avaliar a tolerância dessa simbiose a estas condições.

\section{Material e Métodos}

O experimento foi realizado em casa de vegetação no período de agosto a outubro de 2004. Foram utilizados vasos plásticos contendo $2,8 \mathrm{~kg}$ de um solo classificado como PLANOSSOLO HIDROMÓRFICO Eutrófico arênico com histórico de 22 anos de cultivo de arroz irrigado. $\mathrm{O}$ solo recebeu uma adubação prévia equivalente a $160 \mathrm{~kg} \cdot \mathrm{ha}^{-1}$ de $\mathrm{P}_{2} \mathrm{O}_{5}, 120 \mathrm{~kg} \cdot \mathrm{ha}^{-1}$ de $\mathrm{K}_{2} \mathrm{O}$ e $1000 \mathrm{~kg}$.ha ${ }^{-1}$ de calcário dolomítico. Em cada vaso foram semeadas 10 sementes de trevo branco da variedade Yi. Após a emergência foi realizado um desbaste deixandose apenas quatro plântulas por vaso.

Foi utilizado um delineamento experimental inteiramente casualizado com quatro repetições onde foram avaliados 14 isolados de rizóbio, da coleção da Universidade Federal do Rio Grande do Sul, previamente selecionados de diferentes municípios do estado do Rio Grande do Sul, além das estirpes SEMIA 222 e SEMIA 235, recomendadas para a produção de inoculantes para trevo branco. A inoculação com os isolados e 
estirpes, individualmente, foi realizada logo após a emergência das plântulas, aplicando-se $2 \mathrm{~mL}$ de caldo por vaso, contendo cerca de $10^{9}$ UFC.mL $\mathrm{mL}^{-1}$. Além dos tratamentos inoculados, também foram conduzidos dois tratamentos controles, um sem adição de nitrogênio e outro com a adição de uma dose equivalente a $110 \mathrm{~kg}$. ha ${ }^{1}$ de nitrogênio mineral. Cada tratamento foi mantido sob duas condições de umidade do solo: umidade do solo próxima à capacidade de campo (C.C.) e com alagamento do solo dos 30 aos 60 dias após a inoculação, momento em que foi realizada a colheita do experimento. As avaliações realizadas foram o número e a massa de nódulos secos, a produção de matéria seca e o acúmulo de nitrogênio na parte aérea das plantas (TEDESCO et al., 1995). Os resultados obtidos foram submetidos à análise de variância e ao teste de separação de médias de Duncan $(\mathrm{p}<0,05)$.

\section{Resultados e Discussão}

$\mathrm{O}$ alagamento do solo durante 30 dias, reduziu drasticamente a quantidade de nódulos radiculares nos tratamentos inoculados (Tabela 1). Essa diminuição na quantidade de nódulos radiculares ocasionada pelo alagamento do solo foi observada anteriormente em outros trabalhos (JAMES; CRAWFORD, 1998; VIGNOLIO et al., 1999; SCHOLLES; VARGAS, 2004). Segundo Zhang e Smith (2002), o alagamento do solo causa uma redução significativa no número de sítios de infecção para o rizóbio, diminuindo assim, a quantidade de nódulos radiculares formados.

O alagamento do solo durante 30 dias, reduziu em 33\% a massa de nódulos secos nas plantas dos tratamentos inoculados (Tabela 1). Essa redução média de 33\% na massa de nódulos secos, ocasionada pelo alagamento do solo, também foi observada por Lupwayi et al. (1997) em plantas de Trifolium semipilosum inoculadas com diferentes isolados de rizóbio.

Ao avaliar a viabilidade da inoculação de soja com estirpes de Bradyrhizobium em solo alagado, Scholles e Vargas (2004) observaram que os prejuízos à nodulação, decorrentes do alagamento do solo, foram independentes da estirpe inoculada. Os autores atribuíram esse comportamento a características da planta hospedeira ou à sensibilidade semelhante de todas as quatro estirpes testadas. No entanto, pelo presente trabalho, essa não parece ser uma regra geral, pois tanto na quantidade quanto na massa de nódulos secos, esses prejuízos foram dependentes do rizóbio inoculado (Tabela 1). A redução no número de nódulos causada pelo alagamento do solo variou de $27 \%$ a $61 \%$, observando-se ainda maiores variações na redução da massa de nódulos secos, de $9 \%$ a $58 \%$ dentre os tratamentos inoculados.

TABELA 1: Número e massa de nódulos secos das plantas nos tratamentos em diferentes condições de umidade do solo (médias de quatro repetições).

\begin{tabular}{|c|c|c|c|c|}
\hline \multirow{3}{*}{ Tratamento } & \multicolumn{2}{|c|}{ Número de nódulos ${ }^{1}$} & \multicolumn{2}{|c|}{ Massa de nódulos secos } \\
\hline & C.C. & Alagado & C.C. & Alagado \\
\hline & & & \multicolumn{2}{|c|}{ mg vaso ${ }^{-1}$} \\
\hline PALM 3 & $281 \pm 27^{\mathrm{ab}}$ & $160 \pm 62^{b}$ & $66 \pm 31^{\mathrm{a}}$ & $60 \pm 15^{\mathrm{a}}$ \\
\hline SEMIA 235 & $217 \pm 27^{\mathrm{b}}$ & $159 \pm 33^{b}$ & $79 \pm 9^{a}$ & $60 \pm 17^{\mathrm{a}}$ \\
\hline RP 5 & $376 \pm 149^{\mathrm{ab}}$ & $200 \pm 66^{\mathrm{ab}}$ & $72 \pm 31^{\mathrm{a}}$ & $59 \pm 16^{\mathrm{a}}$ \\
\hline VP 16 & $325 \pm 5^{\mathrm{ab}}$ & $194 \pm 21^{\mathrm{ab}}$ & $76 \pm 14^{\mathrm{a}}$ & $59 \pm 6^{\mathrm{a}}$ \\
\hline N 1 & $479 \pm 238^{\mathrm{a}}$ & $271 \pm 47^{\mathrm{a}}$ & $93 \pm 48^{\mathrm{a}}$ & $57 \pm 14^{\mathrm{ab}}$ \\
\hline HN 18 & $298 \pm 133^{\mathrm{ab}}$ & $181 \pm 18^{\mathrm{ab}}$ & $85 \pm 43^{\mathrm{a}}$ & $53 \pm 7^{\mathrm{abc}}$ \\
\hline $\mathrm{P}_{3} 4$ & $375 \pm 84^{\mathrm{ab}}$ & $193 \pm 32^{\mathrm{ab}}$ & $83 \pm 34^{\mathrm{a}}$ & $52 \pm 8^{\mathrm{abc}}$ \\
\hline $\mathrm{T} 4$ & $301 \pm 43^{\mathrm{ab}}$ & $151 \pm 9^{b}$ & $72 \pm 31^{\mathrm{a}}$ & $52 \pm 4^{\mathrm{abc}}$ \\
\hline SEMIA 222 & $384 \pm 22^{\mathrm{ab}}$ & $148 \pm 18^{b}$ & $98 \pm 51^{\mathrm{a}}$ & $52 \pm 8^{\mathrm{abc}}$ \\
\hline EA 20 & $251 \pm 31^{\mathrm{ab}}$ & $131 \pm 22^{\mathrm{b}}$ & $84 \pm 23^{\mathrm{a}}$ & $52 \pm 11^{\mathrm{abc}}$ \\
\hline SM 3 & $328 \pm 54^{\mathrm{ab}}$ & $198 \pm 23^{\mathrm{ab}}$ & $63 \pm 41^{\mathrm{a}}$ & $48 \pm 12^{\mathrm{abc}}$ \\
\hline CMQ 2 & $274 \pm 30^{\mathrm{ab}}$ & $156 \pm 23^{b}$ & $58 \pm 7^{\mathrm{a}}$ & $46 \pm 9^{\mathrm{abc}}$ \\
\hline CV II & $334 \pm 80^{\mathrm{ab}}$ & $168 \pm 33^{\mathrm{ab}}$ & $69 \pm 9^{a}$ & $42 \pm 8^{\mathrm{bc}}$ \\
\hline AR 20 & $359 \pm 87^{\mathrm{ab}}$ & $147 \pm 14^{b}$ & $82 \pm 36^{\mathrm{a}}$ & $39 \pm 6^{\mathrm{cd}}$ \\
\hline AR 24 & $276 \pm 52^{\mathrm{ab}}$ & $138 \pm 7^{b}$ & $52 \pm 25^{\mathrm{a}}$ & $38 \pm 2^{\mathrm{cd}}$ \\
\hline PF 13 & $350 \pm 112^{\mathrm{ab}}$ & $156 \pm 17^{b}$ & $60 \pm 50^{\mathrm{a}}$ & $25 \pm 4^{\mathrm{d}}$ \\
\hline $\mathrm{T}-\mathrm{N}$ & $20 \pm 38^{c}$ & - & $3 \pm 7^{b}$ & - \\
\hline $\mathrm{T}+\mathrm{N}$ & - & - & - & - \\
\hline
\end{tabular}

Médias seguidas de mesma letra, na coluna, não diferem entre si pelo teste de Duncan a 5\%; ${ }^{1}$ Dados transformados por raiz de $\mathrm{x}+1$.

Assim como a nodulação, a produção de matéria seca da parte aérea das plantas também foi drasticamente reduzida pelo alagamento do solo durante 30 dias, tanto nos tratamentos inoculados quanto nos tratamentos controles, quando comparado aos tratamentos mantidos em umidade do solo próxima à capacidade de campo (Tabela 2). O percentual médio dessa redução foi de $47 \%$, semelhante aos $41 \%$ observados por Lupwayi et al. (1997) em plantas de Trifolium semipilosum inoculadas com diferentes isolados de Rhizobium leguminosarum 
biovar trifolii e mantidas sob alagamento do solo durante 30 dias.

TABELA 2: Produção de matéria seca e acúmulo de nitrogênio na parte aérea das plantas de trevo branco, nos tratamentos inoculados e controle, cultivadas em diferentes condições de umidade do solo (médias de quatro repetições).

\begin{tabular}{|c|c|c|c|c|}
\hline \multirow{3}{*}{ Tratamento } & \multicolumn{2}{|c|}{ Matéria seca } & \multicolumn{2}{|c|}{ Acúmulo de nitrogênio } \\
\hline & C.C. & Alagado & C.C. & Alagado \\
\hline & \multicolumn{2}{|c|}{$\mathrm{g} \mathrm{vaso}^{-1}$} & \multicolumn{2}{|c|}{ Acúmulo de nitrogênio } \\
\hline $\mathrm{T}+\mathrm{N}$ & $3,37 \pm 0,65^{\mathrm{ab}}$ & $2,07 \pm 0,20^{\mathrm{a}}$ & $105,10 \pm 7,92^{\mathrm{a}}$ & $28,10 \pm 1,75^{\mathrm{def}}$ \\
\hline SEMIA 222 & $2,84 \pm 0,79^{\text {abcde }}$ & $1,62 \pm 0,23^{\mathrm{cde}}$ & $60,20 \pm 18,91^{\text {bcde }}$ & $34,10 \pm 3,61^{\mathrm{bcd}}$ \\
\hline SEMIA 235 & $2,72 \pm 0,25^{\text {abcde }}$ & $1,18 \pm 0,13^{\mathrm{f}}$ & $48,60 \pm 6,15^{\mathrm{def}}$ & $23,90 \pm 3,82^{\mathrm{fgh}}$ \\
\hline RP 5 & $3,47 \pm 0,50^{\mathrm{a}}$ & $1,68 \pm 0,15^{\mathrm{bcd}}$ & $64,90 \pm 8,76^{\mathrm{b}}$ & $32,60 \pm 3,46^{\text {cde }}$ \\
\hline $\mathrm{P}_{3} 4$ & $3,17 \pm 0,67^{\mathrm{abc}}$ & $1,84 \pm 0,21^{\mathrm{abc}}$ & $62,60 \pm 9,37^{\mathrm{bc}}$ & $37,50 \pm 3,01^{\mathrm{abc}}$ \\
\hline AR 20 & $2,99 \pm 0,48^{\text {abcde }}$ & $1,51 \pm 0,2^{\mathrm{de}}$ & $62,40 \pm 8,31^{\mathrm{bc}}$ & $32,60 \pm 6,92^{\text {cde }}$ \\
\hline VP 16 & $3,17 \pm 0,18^{\mathrm{abc}}$ & $1,94 \pm 0,15^{\mathrm{ab}}$ & $61,60 \pm 8,54^{\mathrm{bcd}}$ & $40,50 \pm 3,07^{\mathrm{a}}$ \\
\hline $\mathrm{T} 4$ & $2,88 \pm 0,34^{\text {abcde }}$ & $1,83 \pm 0,21^{\mathrm{abc}}$ & $60,10 \pm 5,66^{\text {bcde }}$ & $40,20 \pm 6,05^{\mathrm{ab}}$ \\
\hline CV II & $2,96 \pm 0,15^{\mathrm{abcde}}$ & $1,65 \pm 0,20^{\mathrm{bcd}}$ & $57,90 \pm 8,39$ bcdef & $35,40 \pm 4,80^{\mathrm{abc}}$ \\
\hline PALM 3 & $3,05 \pm 0,16^{\mathrm{abcd}}$ & $1,48 \pm 0,05^{\mathrm{de}}$ & $57,90 \pm 2,97$ bcdef & $28,60 \pm 2,93^{\mathrm{def}}$ \\
\hline CMQ 2 & $2,80 \pm 0,27^{\mathrm{abcde}}$ & $1,53 \pm 0,20^{\mathrm{de}}$ & $53,60 \pm 2,41$ bcdef & $26,50 \pm 2,12^{\mathrm{efg}}$ \\
\hline AR 24 & $2,64 \pm 0,38^{\text {bcde }}$ & $1,52 \pm 0,23^{\mathrm{de}}$ & $53,10 \pm 4,24$ bcdef & $31,20 \pm 5,66^{\text {cde }}$ \\
\hline HN 18 & $2,62 \pm 0,41^{\text {bcde }}$ & $1,55 \pm 0,23^{\mathrm{cde}}$ & $50,70 \pm 8,06^{\text {cdef }}$ & $32,50 \pm 6,71^{\mathrm{cde}}$ \\
\hline N 1 & $2,69 \pm 0,29^{\mathrm{bcde}}$ & $1,33 \pm 0,08^{\mathrm{ef}}$ & $48,70 \pm 8,48$ def & $21,10 \pm 1,54^{\mathrm{gh}}$ \\
\hline SM 3 & $2,85 \pm 0,30^{\text {abcde }}$ & $1,16 \pm 0,09^{\mathrm{f}}$ & $48,60 \pm 5,24$ def & $19,40 \pm 4,43^{\mathrm{h}}$ \\
\hline PF 13 & $2,49 \pm 0,37^{\text {cde }}$ & $0,88 \pm 0,07^{\mathrm{g}}$ & $47,50 \pm 9,09$ ef & $13,00 \pm 1,72^{\mathrm{i}}$ \\
\hline EA 20 & $2,50 \pm 0,68^{\mathrm{cde}}$ & $1,69 \pm 0,22^{\mathrm{bcd}}$ & $44,80 \pm 6,71^{\mathrm{f}}$ & $32,30 \pm 3,70^{\text {cde }}$ \\
\hline
\end{tabular}

T-N $2,23 \pm 0,49^{\circ}$

Médias seguidas de mesma letra, na coluna, não diferem entre si pelo teste de Duncan a 5\%.

Em condições de umidade do solo próximas à capacidade de campo, observou-se que a produção de matéria seca da parte aérea das plantas do tratamento controle com adição de nitrogênio e dos inoculados com os isolados RP5, $\mathrm{P}_{3} 4$, VP16 e PALM3 foi superior à produção de matéria seca do tratamento controle sem adição de nitrogênio (Tabela 2). Já em condições de alagamento do solo, com exceção dos tratamentos inoculados com os isolados SM3, N1, PF13 e com a estirpe SEMIA 235, todos os demais tratamentos inoculados, obtiveram uma produção de matéria seca na parte aérea das plantas superior ao tratamento controle sem adição de nitrogênio (Tabela 2). Observou-se também que as plantas dos tratamentos inoculados com os isolados VP16, $\mathrm{P}_{3} 4$ e T4, obtiveram

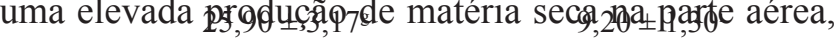
sendo semelhante ao tratamento controle com adição de nitrogênio (Tabela 2).

Da mesma forma que a produção de matéria seca, o acúmulo de nitrogênio na parte aérea das plantas também foi drasticamente reduzido pelo alagamento do solo, com redução média de 49\%. Tais resultados concordam com os observados por Lupwayi et al. (1997), que também observaram redução no acúmulo de nitrogênio na parte aérea das plantas, tanto nos tratamentos inoculados, como nos tratamentos controles com e sem adição de nitrogênio, causada pelo alagamento do solo.

A maior redução no acúmulo de nitrogênio na parte aérea das plantas, em virtude do alagamento, ocorreu no tratamento controle com adição de nitrogênio e no inoculado com o isolado PF13, cerca de 73\%. Essa grande redução no tratamento com adição de nitrogênio, provavelmente ocorreu devido às perdas do nitrogênio 
por desnitrificação após o alagamento do solo. Já no tratamento inoculado com o isolado PF13, essa redução se deve provavelmente a grande sensibilidade desta simbiose às condições de alagamento do solo, indicada pelas elevadas reduções tanto no número $(55 \%)$ e massa de nódulos (58\%) quanto na produção de matéria seca da parte aérea das plantas (65\%). Esse fato é corroborado pelo trabalho de Scholles e Vargas (2004), que observaram diferenças na sensibilidade de estirpes de Bradyrhizobium quanto à fixação simbiótica de nitrogênio em alagamento do solo na cultura da soja.

Nas plantas cultivadas em solo com umidade próxima à capacidade de campo, o maior acúmulo de nitrogênio na parte aérea foi obtido pelas plantas do tratamento controle com adição de nitrogênio. Nessas condições, nenhum dos tratamentos inoculados proporcionou um acúmulo de nitrogênio na parte aérea superior a $62 \%$ do acumulado pelo tratamento controle com adição de nitrogênio (Tabela 2). Esse baixo acúmulo de nitrogênio nas plantas inoculadas com isolados e estirpes, quando comparado ao obtido pelo tratamento controle positivo, possivelmente deve-se ao atraso no início da nodulação e posterior fixação simbiótica do nitrogênio, causado pelo alto teor de nitrogênio mineral no solo no momento de implantação do experimento, cerca de $25 \mathrm{mg} . \mathrm{kg}^{-1}$ solo.

Resultados semelhantes já foram observados em trabalhos anteriores. Avaliando a fixação simbiótica de nitrogênio por isolados de rizóbio em trevo branco, Brose (1994) também observou que nenhum dos tratamentos inoculados, nem mesmo o inoculado com a estirpe SEMIA 222, proporcionou um acúmulo de nitrogênio na parte aérea das plantas semelhante ao tratamento que recebeu a adição de nitrogênio, atribuindo isso, ao efeito negativo do nitrogênio mineral do solo, no processo de fixação simbiótica de nitrogênio.

Um alto teor de nitrogênio mineral no solo pode suprir as necessidades iniciais da planta nesse nutriente, inibindo o processo de infecção e a nodulação pelo rizóbio. Após a absorção de grande parte do nitrogênio mineral do solo, a planta e o rizóbio darão início a simbiose, porém em um momento em que a demanda da planta pelo nutriente é muito superior a demanda no início do ciclo da cultura (STREETER, 1988;
LUCINSKI et al., 2002).

Já em condições de alagamento do solo, os isolados VP16, T4, $\mathrm{P}_{3} 4$ e CVII se mostraram eficientes na fixação simbiótica de nitrogênio quando inoculados em trevo branco. Nessas condições, as plantas inoculadas com esses isolados apresentaram um acúmulo de nitrogênio na parte aérea superior ao obtido no tratamento controle com adição de nitrogênio (Tabela 2), com destaque para o isolado VP16 que proporcionou um acúmulo de nitrogênio na parte aérea superior inclusive ao obtido pelas plantas inoculadas com a estirpe SEMIA 222, indicando um bom potencial desse isolado para fixação simbiótica de nitrogênio em áreas de várzea ou mais sujeitas a alagamentos periódicos.

Esses resultados demonstram a importância da inoculação do trevo branco, principalmente em áreas mais sujeitas ao alagamento, pois nessas condições, é possível que nem mesmo a aplicação de altas doses de nitrogênio mineral garanta um adequado fornecimento de nitrogênio para as plantas, comprometendo assim a qualidade da pastagem e consequentemente o aproveitamento da área.

Na Figura 1, pode-se observar as quantidades de nitrogênio fixado na parte aérea das plantas inoculadas em alagamento do solo, em relação às obtidas em capacidade de campo, o que permite avaliar a sensibilidade da simbiose ao alagamento do solo. Observa-se que a inoculação do isolado PF13 em alagamento do solo proporcionou apenas $18 \%$ da quantidade de nitrogênio fixada em condições de umidade do solo próximas à capacidade de campo (Figura 1), confirmando a elevada sensibilidade desse isolado quanto à sua eficiência na fixação simbiótica de nitrogênio, às condições de alagamento do solo.

Por outro lado, no tratamento inoculado com o isolado EA20, a quantidade de nitrogênio fixada foi maior em condições de alagamento do solo do que em condições de umidade do solo próximas à capacidade de campo, evidenciado pelo valor de $123 \%$ de aproveitamento relativo (Figura 1). Isto indica que o alagamento do solo não afetou negativamente e, de alguma forma, até beneficiou o processo de fixação simbiótica de nitrogênio. 
FIGURA 1: Aproveitamento relativo da fixação simbiótica de nitrogênio nas condições de alagamento do solo em relação à fixação nas condições de umidade do solo próximas à capacidade de campo.

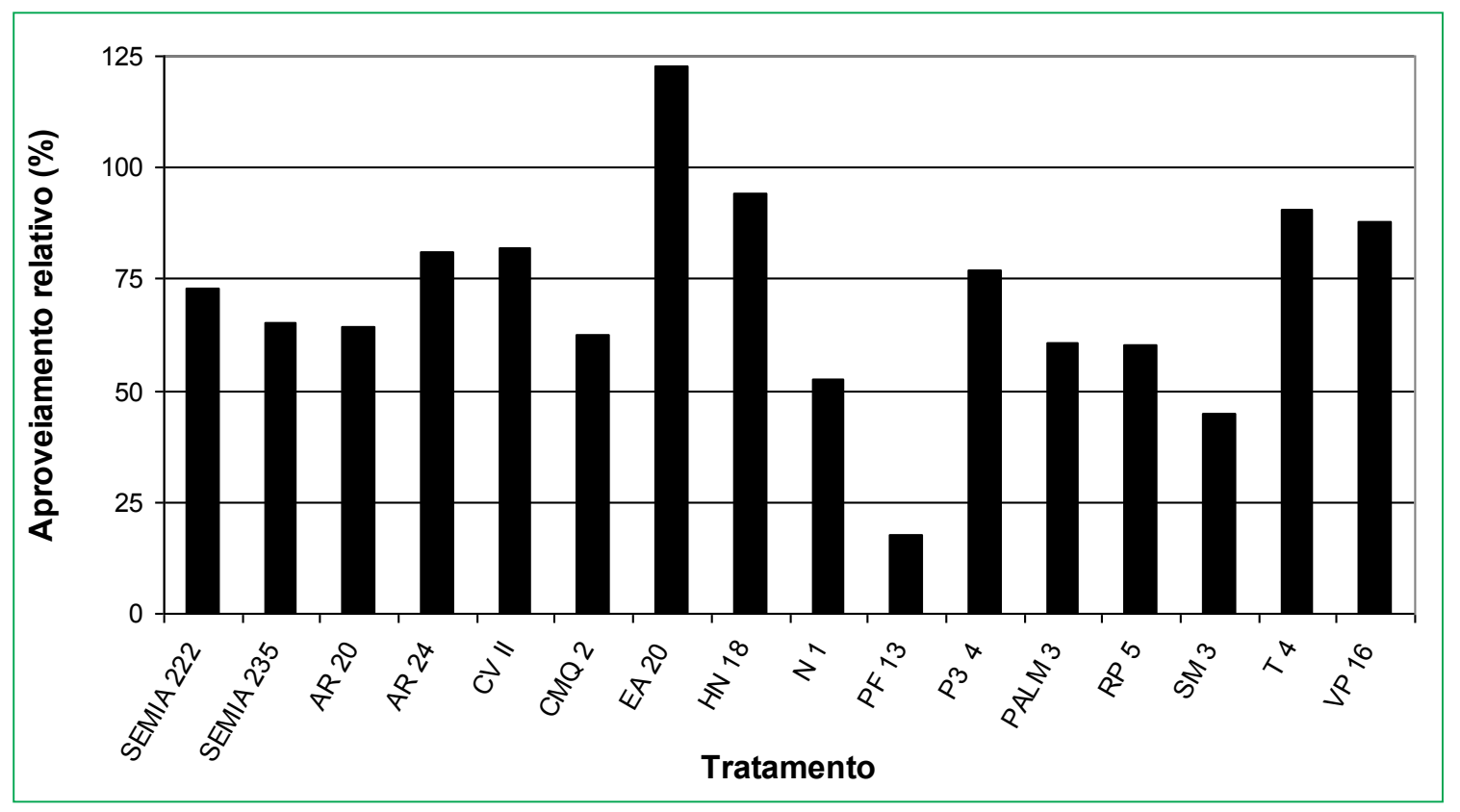

Esses resultados demonstram que em condições de alagamento do solo, a eficiência na FBN pela simbiose rizóbio/trevo branco é bastante variável em função da maior ou menor sensibilidade de cada rizóbio a essas condições. Isso mostra importância de se levar em consideração essas características no momento de definir recomendações de inoculação em diferentes situações, como em áreas mais sujeitas ao alagamento.

A partir dos resultados, foi possível concluir que:

1) O alagamento do solo afeta a simbiose rizóbio/trevo branco reduzindo o número e a massa de nódulos secos, assim como o acúmulo de nitrogênio e a produção de matéria seca da parte aérea das plantas de trevo branco;

2) A fixação de nitrogênio pela simbiose rizóbio/trevo branco pode ocorrer em condições de alagamento do solo como as que ocorrem em áreas de várzea;

3) Existe variabilidade na sensibilidade da simbiose rizóbio/trevo ao alagamento do solo em função do rizóbio inoculado;

4) Os isolados VP16, T4, $P_{3} 4$ e CVII são eficientes na fixação simbiótica de nitrogênio com trevo branco em condições de solo alagado, constituindo-se em um material promissor para programas de seleção de estirpes para trevo branco.

\section{Referências}

BROSE, E. Seleção de rizóbio para trevo-branco em solo ácido. Pesquisa Agropecuária Brasileira, Brasília, v. 29, n. 2, p. 281285, 1994.

CARLSSON, G.; HUSS-DANELL, K. Nitrogen fixation in perennial forage legumes in the field. Plant and Soil, New York, v. 253 , n. 2, p. 353-372, 2003.

GIBBERD, M. R.; GRAY, J. D.; COCKS, P. S.; COLMER, T. D. Waterlogging tolerance among a diverse range of Trifolium accessions is related to root porosity, lateral root formation and "aerotropic rooting". Annals of Botany, Oxford, v. 88, p. 579-589, 2001.

JAMES, E. K.; CRAWFORD, R. M. M. Effect of oxygen availability on nitrogen fixation by two Lotus species under flooded conditions. Journal of Experimental Botany, Oxford, v. 49, n. 320, p. 599-609, 1998.

JAMES, E. K.; SPRENT, J. I. Development of $\mathrm{N}_{2}$-fixing nodules on the wetland legume Lotus uliginosus exposed to conditions of flooding. New Phytologist, Oak Ridge, v. 142, p. 219-231, 1999.

LUCINSKI, R.; POLCYN, W.; RATAJCZAK, L. Nitrate reduction and nitrogen fixation in symbiotic association Rhizobium - legumes. Acta Biochimica Polonica, Warsaw, v. 49, n. 2, p. 537-546, 2002. LUPWAYI, N. Z.; HAQUE, I.; HOLL, F. B. Strain-specific response of Trifolium semipilosum to inoculation with Rhizobium and the 
significance of waterlogging in Vertisols. Journal of Agricultural Science, Cambridge, v. 129, p. 439-446, 1997.

MARCHEZAN, E.; VIZZOTTO, V. R.; ROCHA, M. G.; MOOJEN, E. L.; SILVA, J. H. S. Produção animal em várzea sistematizada cultivada com forrageiras de estação fria submetidas a diferentes níveis de adubação. Ciência Rural, Santa Maria, v. 32, p. 303-308, 2002.

PUGH, R.; WITTY, J. F.; MYTTON, L. R.; MINCHIN, F. R. The effect of waterlogging on nitrogen fixation and nodule morphology in soil-grown white clover (Trifolium repens L.). Journal of Experimental Botany, Oxford, v. 46, p. 285-290, 1995.

ROGERS, M. E.; WEST, D. W. The effects of rootzone salinity and hypoxia on shoot and root growth in Trifolium species. Annals of Botany, Oxford, v. 72, p. 503-509, 1993.

SCHOLLES, D.; VARGAS, L. K. Viabilidade da inoculação de soja com estirpes de Bradyrhizobium em solo inundado. Revista Brasileira de Ciência do Solo, Viçosa, v. 28, n. 6, p. 973-979, 2004.
STREETER, J. Inhibition of legume nodule formation and $\mathrm{N}_{2}$ fixation by nitrate. CRC Critical Reviews in Plat Sciences, Apopka, v. 7, p. 1-23, 1988.

TEDESCO, M. J.; GIANELO, C. BISSANI, C. A. Análise de solo, plantas e outros materiais. 2. ed. Porto Alegre: Departamento de Solos da UFRGS, 1995. 174 p. (Boletim técnico, 5).

VARTAPETIAN, B. B.; JACKSON, M. B. Plant adaptations to anaerobic stress. Annals of Botany, Oxford, v. 79, p. 3-20, 1997.

VIGNOLIO, O. R.; FERNÁNDEZ, O. N.; MACEIRA, N. O. Flooding tolerance in five populations of Lotus glaber Mill. (Syn. Lotus tenuis Waldst. Et. Kit.). Australian Journal of Agricultural Research, East Melbourne, v. 50, p. 555-559, 1999.

ZHANG, F.; SMITH, D.L. Interorganismal signaling in suboptimum environments: The legume-rhizobia symbiosis. Advances in Agronomy, San Diego, v. 76, p. 125-161, 2002. 